\title{
Fine particulate matter exposure promotes M2 macrophage polarization through inhibiting histone deacetylase 2 in the pathogenesis of chronic obstructive pulmonary disease
}

\author{
Yan Jiang", Yanfeng Zhao", Qingliang Wang, Hao Chen, Xiao Zhou \\ Department of Thoracic Surgery, Shanghai Pulmonary Hospital, Tongji University School of Medicine, Shanghai, China \\ Contributions: (I) Conception and design: X Zhou, Y Jiang; (II) Administrative support: X Zhou; (III) Provision of study materials or patients: All \\ authors; (IV) Collection and assembly of data: All authors; (V) Data analysis and interpretation: All authors; (VI) Manuscript writing: All authors; (VII) \\ Final approval of manuscript: All authors. \\ \#These authors contributed equally to this work. \\ Correspondence to: Xiao Zhou. Department of Thoracic Surgery, Shanghai Pulmonary Hospital, Tongji University School of Medicine, Shanghai \\ 200433, China. Email: zx_ty68@163.com.
}

\begin{abstract}
Background: Chronic obstructive pulmonary disease (COPD) is a major cause of morbidity and mortality globally. Fine particulate matter (PM2.5) has been indicated to be a major detrimental risk factor for COPD by numerous epidemiological studies. Histone deacetylase 2 (HDAC2), a critical regulator of chromatin remodeling, plays a pivotal role in the development of COPD. However, the underlying mechanisms regarding the relationship between PM2.5 and HDAC2 in the pathogenesis of COPD have yet to be elucidated. In the present study, we aim to investigate the role and the underlying mechanism of HDAC2 in the development of PM2.5-induced COPD.

Methods: The effects of PM2.5 exposure on M2 macrophage polarization and the expression levels of HDAC2 were examined in vitro. The influence of HDAC2 deficiency on M2 macrophage polarization and the pathogenesis of COPD was investigated in a PM2.5-induced mouse model.

Results: PM2.5 exposure down-regulated the protein level of HDAC2 and enhanced M2 macrophage polarization in vitro. In the COPD murine model, myeloid-specific deficiency of HDAC2 augmented PM2.5-induced M2 polarization of alveolar macrophages (AMs) and up-regulation of tumor necrosis factor (TGF)- $\beta$, matrix metallopeptidase (MMP)-9, and MMP-12 in lung tissue, which resulted in more prominent lung function deterioration, airspace enlargement, alveolar wall destruction, and airway remodeling, indicating a key role of HDAC2 in the pathogenesis of PM2.5-induced COPD.

Conclusions: PM2.5 facilitated M2 polarization by inhibiting HDAC2, leading to the development of COPD. Targeting of HDAC2 would provide a novel approach to prevent the development of PM2.5 exposure-induced COPD.
\end{abstract}

Keywords: PM2.5; M2 macrophage polarization; histone deacetylase 2 (HDAC2); chronic obstructive pulmonary disease (COPD)

Submitted Jul 31, 2020. Accepted for publication Oct 12, 2020.

doi: 10.21037/atm-20-6653

View this article at: http://dx.doi.org/10.21037/atm-20-6653

\section{Introduction}

Chronic obstructive pulmonary disease (COPD) is a major cause of global morbidity and mortality that is characterized by irreversible and progressive obstruction of the airways. It is predicted that COPD would be the third leading cause of death worldwide by $2030(1,2)$.

Over the past decades, ambient fine particulate matter (PM2.5) exposure has been described as a major detrimental 
risk factor for COPD in numerous epidemiological studies (3-5). In COPD patients, PM2.5 has been found to not only be associated with exacerbated and aggravated respiratory function and symptoms (6) but also with increased risk morbidity and mortality $(7,8)$.

Macrophages are a crucial component of innate immunity, representing the first line of defense against extrinsic invaders (9). In the lung, there are at least two distinct macrophage populations: alveolar macrophages (AMs) and interstitial macrophages (IMs). Comprising more than $90 \%$ of the pulmonary macrophage population, AMs play a central role in defending the lung against airborne irritants and pathogens (10).

Macrophages adapt and respond to a variety of environmental stimuli, exhibiting remarkable plasticity. Macrophages may undergo classical activation (M1 macrophages) or alternative activation (M2 macrophages) depending on the environmental signals $(11,12)$. The M1 phenotype is induced by granulocyte-macrophage colonystimulating factor (GM-CSF), lipopolysaccharide (LPS), or interferon- $\gamma(\mathrm{IFN}-\gamma)$, and is characterized by a high expression level of nitric-oxide synthase (iNOS) and the secretion of pro-inflammatory cytokines, such as tumor necrosis factor- $\alpha$ (TNF- $\alpha$ ), interleukin (IL)-1 $\beta$, IL- 6 , and IL-12; thus, M1 macrophages are mainly involved in proinflammatory responses and cytotoxic effects $(11,13-15)$. M2 macrophages are stimulated by monocyte colonystimulating factor (M-CSF), IL-4, IL-13 and IL-10, and are typified by a high expression level of arginase-1 (Arg-1) and the secretion of cytokines including IL-4, IL-10, and TGF- $\beta$. The M2 phenotype also has anti-inflammatory properties and is involved in tissue remodeling and fibrosis (14-17). An imbalance of M1/M2 polarization can result in the development of inflammatory diseases, including COPD $(9,18)$.

Histone deacetylase 2 (HDAC2), as one of the members of the class I HDACs, plays a critical regulatory role in gene expression (19). Previous studies have reported that HDAC2 levels and activity are reduced in patients with COPD (20), which may contribute to chronic inflammation and subsequent remodeling and obstruction of the airways. This suggests that HDAC2 has a critical role in the development of COPD. Despite clear evidence of HDAC2's involvement in mediating lung damage in established COPD, its role in the regulation of macrophage polarization during the process of COPD development is not fully understood.

In the present study, we aimed to determine whether HDAC2 is involved in the development of PM2.5- induced COPD through regulating the balance of M1/ M2 macrophages. The effects of PM2.5 exposure on the expression levels of HDAC2 and macrophage polarization were analyzed in vitro. The influence of HDAC2 deficiency on macrophage polarization and the pathogenesis of COPD was investigated in a PM2.5-induced COPD mouse model.

We present the following article in accordance with the ARRIVE reporting checklist (available at http://dx.doi. org/10.21037/atm-20-6653).

\section{Methods}

\section{PM2.5 collection and extraction}

PM2.5 was continuously collected using an air sampler (LB-6120, Loobo, Qindao, China) with Teflon microporous membrane filters. The samples were collected from ambient air near a road close to Shanghai Pulmonary Hospital at a flow rate of $300 \mathrm{~L} / \mathrm{min}$. After sampling, the filters were placed in petri dishes and stored at $-20^{\circ} \mathrm{C}$. Then, the PM2.5 particulates were removed from the filter and immersed in sterile purified $\mathrm{H}_{2} \mathrm{O}$. This was followed by ultrasonication for $4 \times 30 \mathrm{~min}$. After drying by lyophilisation, the PM2.5 powder was stored at $-20^{\circ} \mathrm{C}$ until use.

\section{Induction of the COPD model}

$\mathrm{HDAC}^{\mathrm{A} / \mathrm{f}}$ and Lys2-Cre mice (C57BL/6 background) were supplied by Shanghai Model Organisms. Myeloid-specific HDAC2 knockout mice (HDAC2 CKO) were generated by crossing $\mathrm{HDAC}^{\mathrm{fl} / \mathrm{fl}}$ mice with Lys2-Cre mice. The HDAC2 CKO and HDAC2 ${ }^{\mathrm{A} / \mathrm{t}}$ mice were randomly divided into the PM2.5-exposed group and the control group ( $\mathrm{n}=6$ per group). The PM2.5-exposed mice were continuously subjected to whole-body inhalation of concentrated ambient air PM2.5 in vivo for 30 weeks. Simultaneously, the control group mice were exposed to filtered air. All animal experimental procedures were approved by the Ethics Committee for Animal Studies at Shanghai Pulmonary Hospital affiliated to Tongji University (No. K19-100Y) and were carried out in accordance with institutional conventions for the care and use of animals.

\section{Measurement of pulmonary function}

Pulmonary function was measured by using the AniRes 2005 lung function system (Bestlab, Beijing, China). The mice were anaesthetized via intraperitoneal injection of 
$10 \%$ chloral hydrate and then tracheostomized. The mice were immediately placed in the supine position in a sealed whole-body plethysmograph and connected to a computercontrolled small animal ventilator via the tracheal cannula. All animals were mechanically ventilated at a respiratory rate of 90 breaths $/ \mathrm{min}$ with a tidal volume of $5 \mathrm{~mL} / \mathrm{kg}$ and an expiration/inspiration time ratio of 1.5:1.0. Forced expiratory vital capacity (FVC), forced expiratory volume in $0.1 \mathrm{~s}\left(\mathrm{FEV}_{0.1}\right)$, and the $\mathrm{FEV}_{0.1} / \mathrm{FVC}$ ratio were measured.

\section{Collection of bronchoalveolar lavage fuid}

The mice were sacrificed at the end of week 30 . The chests of the mice were opened, and the tracheas were intubated with a tracheal cannula. Before bronchoalveolar lavage fluid (BALF) was collected, the left main bronchus was ligated to prevent operations from impacting on pulmonary morphometric and pathological assessments. Then, the BALF was collected by injecting $1 \mathrm{ml}$ of phosphate-buffered saline (PBS) into the right lung. The collected BALF was filtered with gauze and centrifuged at 1,200 rpm for $5 \mathrm{~min}$ at $4{ }^{\circ} \mathrm{C}$. Cells were washed twice with PBS and suspended in Dulbecco's Modified Eagle Medium (DMEM) containing $10 \%$ fetal bovine serum (FBS), $100 \mathrm{U} / \mathrm{mL}$ penicillin, and $100 \mathrm{U} / \mathrm{mL}$ streptomycin. For quantitative reverse transcription- polymerase chain reaction (qRT-PCR), the remaining cells were seeded in 6 -well culture dishes $\left(1 \times 10^{6} \%\right.$ well), and the AMs were incubated for $2 \mathrm{~h}$ in a $5 \% \mathrm{CO}_{2}$ humidified incubator. Finally, the cells were washed twice with PBS to remove non-adherent cells.

\section{Pulmonary pathology assessment}

The right lung of the mice was excised, fixed in $4 \%$ paraformaldehyde, and then embedded in paraffin. Lung tissue was stained with hematoxylin and eosin (H\&E) and examined under light microscopy for histologic and morphometric examination.

\section{Isolation and stimulation of bone marrow-derived macrophages}

To obtain bone marrow-derived macrophages (BMDMs), femur and tibia bones were collected from 5 -week-old mice, and the bone marrow cells were flushed out using PBS supplemented with $2 \%$ FBS. Red blood cells were lysed with $\mathrm{NH}_{4} \mathrm{Cl}$ solution, and the remaining cells were cultured in DMEM medium containing 100 units $/ \mathrm{mL}$ penicillin and
$100 \mu \mathrm{g} / \mathrm{mL}$ streptomycin, supplemented with $10 \%$ FBS. The cells were maintained in a humidified atmosphere of $5 \%$ $\mathrm{CO}_{2}$ at $37^{\circ} \mathrm{C}$. For analysis of M2 macrophage polarization, cells were stimulated with $10 \mathrm{ng} / \mathrm{ml}$ GM-CSF and IL-4.

\section{qRT-PCR analysis}

Total RNA was extracted using TRIzol reagent (Invitrogen, USA) and reverse transcribed into cDNA using EasyScript ${ }^{\circledR}$ Fist-Strand cDNA Synthesis Supermix (Transgen, China). The levels of RNA were determined using TransStart ${ }^{\circledR}$ Green qPCR Supermix (Transgen, China) on a 7900HT qRT-PCR machine (Applied Biosystems). The relative levels of messenger RNA (mRNA) were analyzed using the $\Delta \Delta \mathrm{Ct}$ method.

\section{Western blot}

Cells were lysed with RIPA lysis buffer (Beyotime Biotech, China). The protein concentration was determined by BCA protein assay kit (Tiangen Biotech, China). Equal amounts of protein were loaded onto $10 \%$ sodium dodecyl sulfate-polyacrylamide gel electrophoresis (SDS-PAGE) gel and transferred onto a polyvinylidene fluoride (PVDF) membrane by electrophoresis. The membrane was then incubated with anti-HDAC2 (Abcam, Cambridge, UK)), anti- $\beta$-actin (CST, USA), and horseradish peroxidase (HRP)-conjugated goat anti-rabbit IgG (CST, USA). The HRP ECL Chemiluminescent Substrate Kit (ThermoFisher Scientific, USA) was used for color development.

\section{Flow cytometry}

Activated BMDMs or freshly isolated AMs were washed with PBS and re-suspended in PBS containing $1 \%$ bovine serum albumin (BSA). Subsequently, cells were stained with anti-CD206 (eBiosciences, USA), incubated at room temperature for $30 \mathrm{~min}$, and then fixed, permeated, and stained with anti-Arg-1 (eBiosciences, USA) for $1 \mathrm{~h}$. Finally, the cells were washed with PBS, and analyzed with the fluorescence-activated cell sorting (FACS) Calibur system (BD Biosciences, San Diego, CA, USA).

\section{Statistical analysis}

Data were expressed as mean \pm standard deviation (SD). All statistical analyses were performed with GraphPad Prism 7.0 software (GraphPad Software, Inc). Data were analyzed 
A

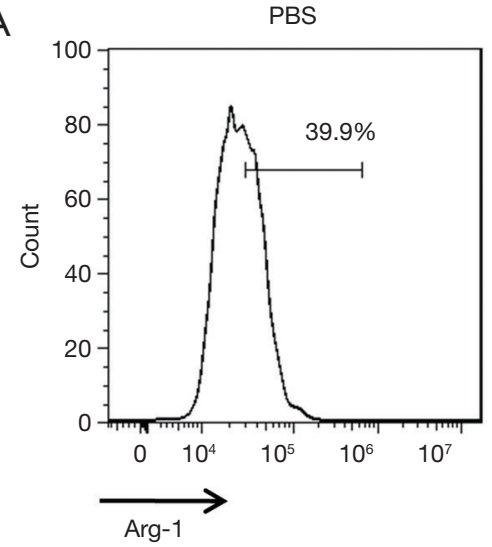

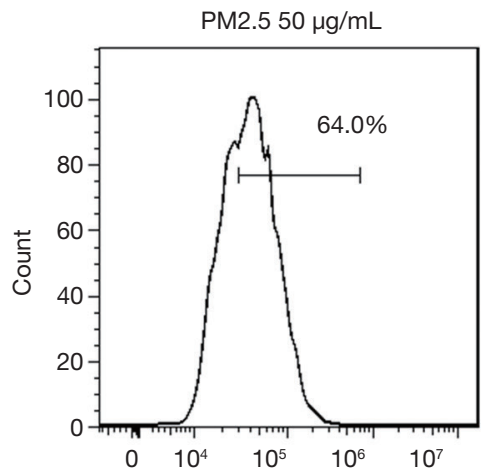

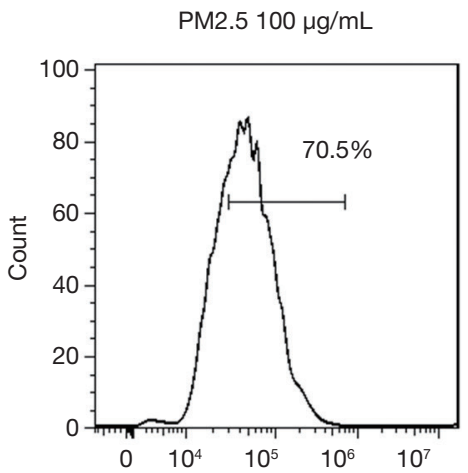

B

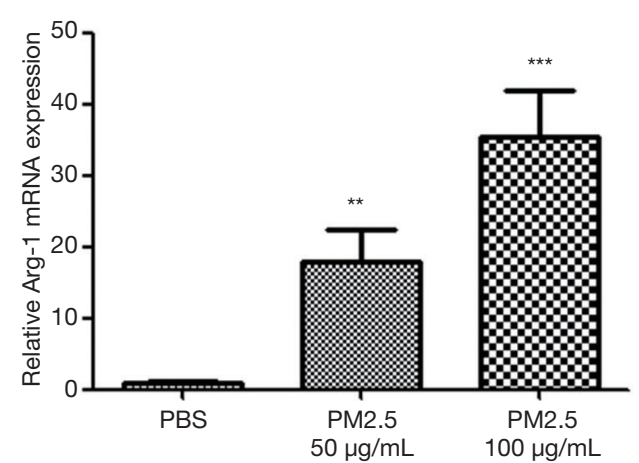

Figure 1 PM2.5 facilitated M2 macrophage polarization in vitro. M2 polarization of BMDMs was induced by $10 \mathrm{ng} / \mathrm{mL}$ GM-CSF and IL-4 following treatment with PBS or PM2.5 (50 or $100 \mu \mathrm{g} / \mathrm{mL}$ ). (A) Representative FACS plots of M2 macrophages. (B) The mRNA expression levels of the M2 macrophage marker Arg-1. **, $\mathrm{P}<0.01$; ***, $\mathrm{P}<0.001$. PM2.5, fine particulate matter; BMDMs, bone marrowderived macrophages; GM-CSF, granulocyte-macrophage colony-stimulating factor; IL, interleukin; PBS, phosphate-buffered saline; FACS, fluorescence-activated cell sorting.

using the Student's $t$-test for comparison between two groups or one-way ANOVA test for multiple comparisons. $\mathrm{P}<0.05$ was considered to be statistically significant.

\section{Results}

\section{PM2.5 facilitated M2 polarization in vitro}

To examine the impact of PM2.5 on macrophage polarization, BMDMs were incubated with PM2.5 (50 or $100 \mu \mathrm{g} / \mathrm{mL}$ ) or PBS for $24 \mathrm{~h}$ before the induction of M2 polarization. The efficiency of M2 polarization of BMDMs was determined by FACS, and the expression level of Arg1 mRNA was examined by qRT-PCR. PM2.5 was observed to enhance M2 polarization in a dose-dependent manner, as evidenced by an increase in the ratio of Arg-1-positive BMDMs (Figure $1 A$ ) and the augmented mRNA expression level of Arg-1 (Figure 1B).

\section{PM2.5 down-regulated the protein expression level of HDAC2}

To investigate the mechanism by which PM2.5 interferes with $\mathrm{M} 2$ macrophage polarization, we examined the influence of PM2.5 on the expression level of HDAC2, which is reported to be down-regulated in the PBMCs of COPD patients (21). HDAC2 protein expression was examined by Western blot, and HDAC2 mRNA expression level was quantified by qRT-PCR. As shown in Figure 2, the protein expression of HDAC2 in BMDMs was dose-dependently reduced by exposure to PM2.5, while the mRNA expression level was not significantly changed. These results suggested PM2.5 may affect the degradation process of HDAC2 in a manner similar to cigarette smoke (CS) exposure. 

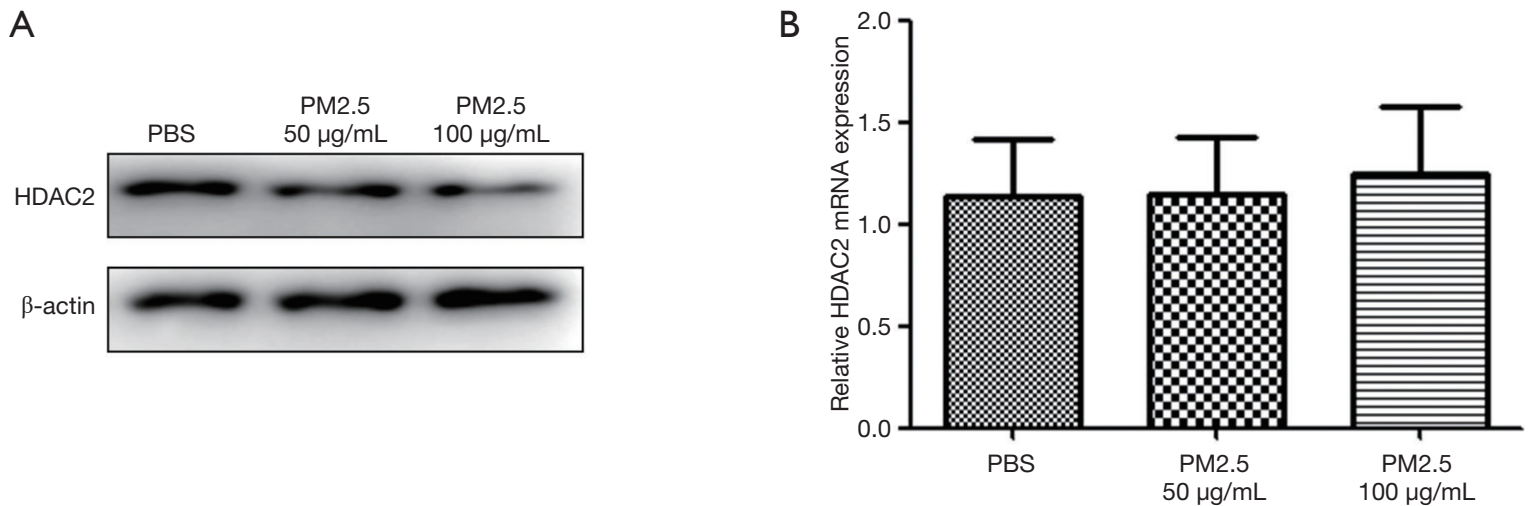

Figure 2 PM2.5 down-regulated the protein expression level of HDAC2 in vitro. BMDMs were incubated with PM2.5 or PBS for 72 h. Then, the level of HDAC2 protein expression was detected with Western blot (A). The mRNA expression level of HDAC2 was detected by qRT-PCR (B). PM2.5, fine particulate matter; HDAC2, histone deacetylase 2; PBS, phosphate-buffered saline; qRT-PCR, quantitative reverse transcription- polymerase chain reaction.

\section{HDAC2 deficiency facilitated M2 polarization in vitro}

To investigate whether HDAC2 plays a role of in the alternative activation of macrophage, we explored the effect of HDAC2 deficiency on M2 macrophage polarization in BMDMs. The M2 polarization efficiency of BMDMs was determined by FACS, and the expression level of Arg-1 mRNA was examined by qRT-PCR. The ratio of Arg-1positive BMDMs was increased and the mRNA expression level of Arg-1 was augmented, which showed that HDAC2 deficiency facilitated M2 polarization (Figure $3 A, B$ ).

\section{HDAC2 deficiency exacerbated the decline of lung function, airspace enlargement, and airway remodeling in the PM2.5 induced COPD mouse model}

To determine whether HDAC2 is functionally linked to the pathogenesis of PM2.5-induced COPD, we compared the tendency of WT and HDAC2 CKO mice to develop COPD. The development of COPD was simulated by exposing mice to PM2.5 for 30 weeks. As shown in Table 1, a decline in $\mathrm{FEV}_{0.1} / \mathrm{FVC}$, decreased $\mathrm{FEV}_{0.1}$, and increased FVC were observed in the HDAC2 CKO group. These results indicated that PM2.5 triggered a more severe decline in pulmonary function in the HDAC2 CKO mice than in the $\mathrm{HDAC}_{2}^{\mathrm{A} / \mathrm{fl}}$ mice. The results of H\&E staining of mouse lung sections were consistent with this finding, with exacerbated enlargement of the alveolar space, alveolar wall destruction, and airway remodeling, as well as inflammatory cell infiltration, observed in the HDAC2 CKO mice (Figure 4).

\section{HDAC2 deficiency facilitated macrophage alternative activation in the PM2.5-induced COPD mouse model.}

The effect of HDAC2 deficiency on M2 polarization in the PM2.5-induced COPD mouse model was investigated. The percentage of M2 macrophages in the BALF of each group was examined by FACS. The expression levels of MMP9, MMP-12, and TGF- $\beta 1$ were determined by qRT-PCR. BALF from the HDAC2 CKO mice showed a marked increase in the percentage of $\mathrm{M} 2$ macrophages compared to that from the $\mathrm{HDAC}^{\mathrm{A} / \mathrm{f}}$ mice (Figure $5 A, B$ ), with significant increases in the M2-related cytokines MMP-9, MMP-12, and TGF- $\beta$ (Figure 5C), which led to enhanced remodeling of airway.

\section{Discussion}

COPD is a chronic progressive inflammatory disease that has a rising prevalence worldwide (2). The chronic nature of COPD, and the exacerbations and comorbidities it frequently entails, impose a heavy clinical and economic burden on society. Therefore, the exploration of novel therapeutic options for COPD is warranted (22). There is a growing body of evidence to associate PM2.5 exposure with increased morbidity and mortality, as well as the pathogenesis of COPD. However, the underlying mechanisms by which PM2.5 exposure induces and aggravates the development of COPD remain unclear.

In this study, we found that PM2.5 exposure facilitates M2 polarization state in vitro and in vivo. M2 polarization enables macrophages to play a role in the regulation 

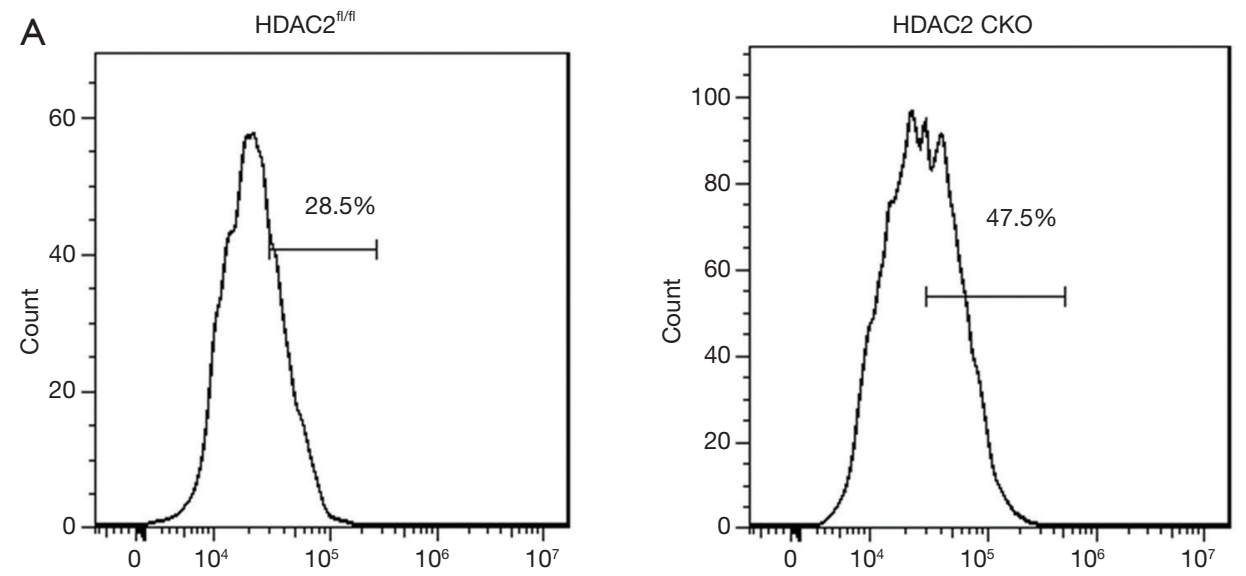

B

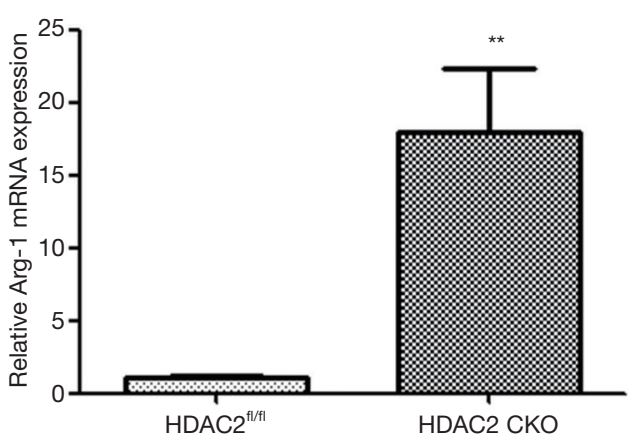

Figure 3 HDAC2 deficiency facilitated M2 macrophage polarization in vitro. M2 polarization of BMDMs was induced by 10 ng/mL GMCSF and IL-4 following treatment with $100 \mu \mathrm{g} / \mathrm{mL}$ PM2.5. (A) Representative FACS plots of M2 macrophages. (B) The mRNA expression level of the M2 macrophage marker Arg-1. **, P<0.01. HDAC2, histone deacetylase 2; BMDMs, bone marrow-derived macrophages; GMCSF, granulocyte-macrophage colony-stimulating factor; IL, interleukin; PM2.5, fine particulate matter.

Table 1 Pulmonary function in each group

\begin{tabular}{llll}
\hline Group & FEV $_{0.1} / \mathrm{FVC}$ & $\mathrm{FEV}_{0.1}(\mathrm{~mL})$ & $\mathrm{FVC}(\mathrm{mL})$ \\
\hline HDAC2 $^{\text {fl/fl }}+$ air & $82.44 \pm 2.53$ & $0.77 \pm 0.09$ & $1.03 \pm 0.12$ \\
HDAC2 CKO + air & $83.73 \pm 3.02$ & $0.76 \pm 0.07$ & $1.21 \pm 0.12$ \\
HDAC2 $^{\text {fl/fl }}+$ PM2.5 & $64.42 \pm 4.98$ & $0.73 \pm 0.11$ & $1.11 \pm 0.15$ \\
HDAC2 CKO + PM2.5 & $50.35 \pm 5.13^{*}$ & $0.43 \pm 0.05^{\star}$ & $0.79 \pm 0.05$ \\
\hline
\end{tabular}

Values are mean $\pm \mathrm{SD}, \mathrm{n}=6$ mice per group. ${ }^{*} \mathrm{P}<0.05$ compared to the HDAC2 ${ }^{\mathrm{f} / \mathrm{fl}}+\mathrm{PM} 2.5$ group. FVC, forced expiratory vital capacity; $\mathrm{FEV}_{0.1}$, forced expiratory volume in $0.1 \mathrm{~s}$; HDAC2, histone deacetylase 2; PM2.5, fine particulate matter.

of tissue repair and airway remodeling (17). From this observation, an important question arises as to how PM2.5 interferes with the macrophage polarization program. Numerous studies have shown that reduced HDAC2 expression and activity in COPD patients is secondary to the posttranslational modifications, which include phosphorylation, oxidation, ubiquitination, and subsequent degradation of HDAC2 in response to tobacco exposure $(23,24)$. In this study, the level of HDAC2 protein expression in BMDMs was reduced by PM2.5 exposure, while the mRNA level was not significantly changed, which suggests that PM2.5 may also affect the degradation process of HDAC2 in a similar manner to CS exposure. Glucocorticoids which reverse the histone 

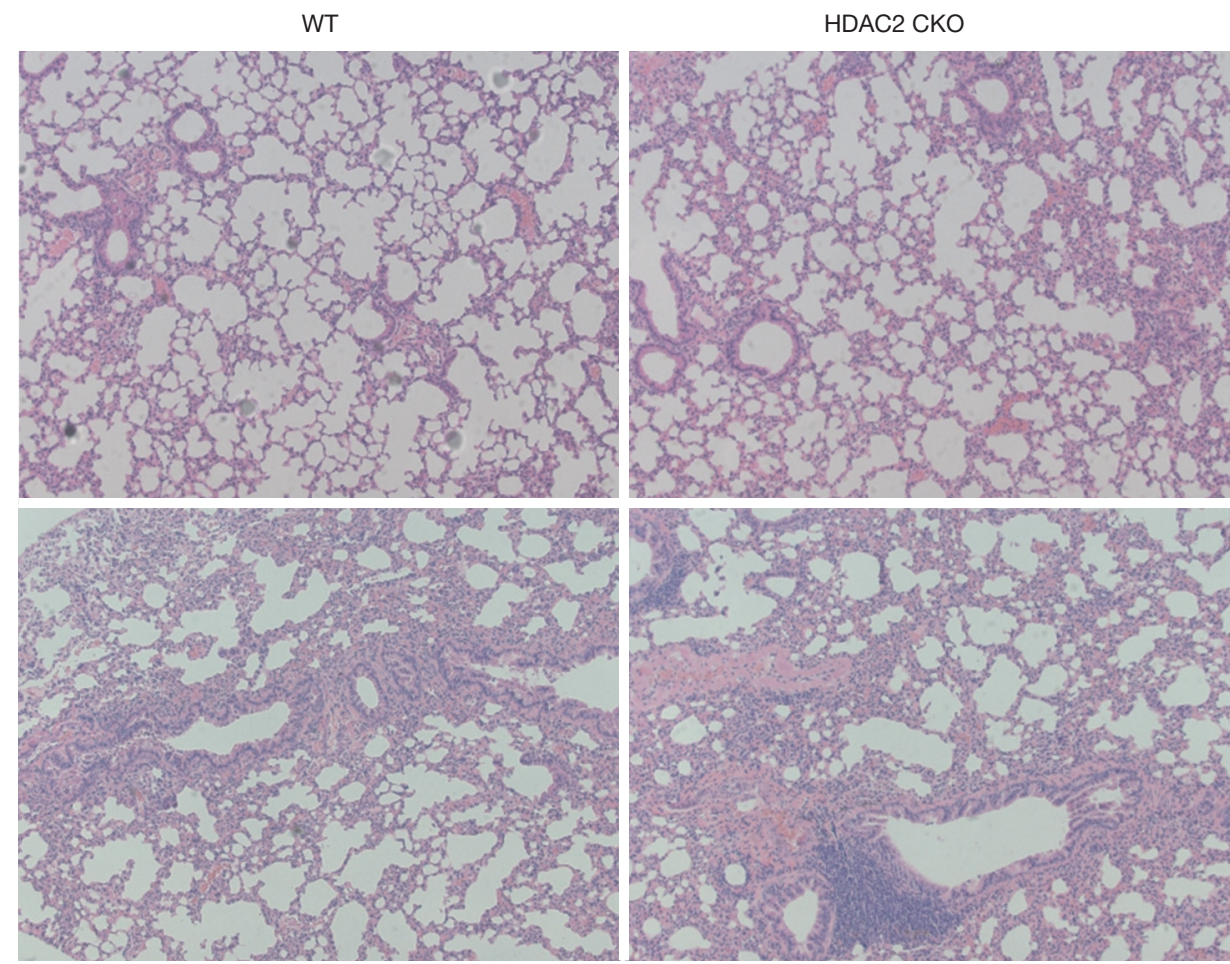

Air

PM2.5

Figure 4 HDAC2 deficiency led to worsened lung histomorphology in a PM2.5-induced COPD model. H\&E staining of lung tissue (100× magnification) shows enlargement of the alveolar space, alveolar wall destruction, and airway remodeling, as well as inflammatory cell infiltration. PM2.5, fine particulate matter; HDAC2, histone deacetylase 2; COPD, chronic obstructive pulmonary disease.

acetylation induced by nuclear factor- $\kappa \mathrm{B}(\mathrm{NF}-\kappa \mathrm{B})$ and switches off the activated inflammatory genes by binding to glucocorticoid receptors (GR) and recruiting HDAC2 to the activated inflammatory genes, are very effective in controlling the airway inflammation in asthma $(25,26)$. However, due to the reduction in HDAC2 expression in patients with COPD, corticosteroid provide little clinical benefit in this population. Understanding the mechanism of action of PM2.5 in reducing HDAC2 expression may help reverse and bypass corticosteroid resistance in COPD patients.

An important feature of the pathogenesis of COPD is persistent airflow limitation due to progressive parenchymal destruction and the remodeling of peripheral airways (4). An imbalance between proteinases and their inhibitors is pivotal in alveolar destruction and airway remodeling during COPD development (27). Furthermore, TGF- $\beta 1$ also plays an essential role in lung remodeling $(28,29)$. Our study showed that long-term PM2.5 exposure enhanced the induction of $\mathrm{M} 2$ polarization and up-regulation of TGF- $\beta$, MMP-9, and MMP-12 in HDAC2 CKO mice compared with $\mathrm{HDAC}^{\mathrm{fl} / \mathrm{fl}}$ mice, which led to more significant airspace enlargement, alveolar wall destruction, and airway remodeling. These results indicate that HDAC2 plays a key role in the pathogenesis of COPD. Targeting HDAC2 to release the brake on alternative activation would provide a novel approach to prevent the progression of COPD. However, whether the function of HDAC2 in ameliorating M2 activation is dependent on HDAC activity still needs to be determined. Future studies to validate this and to identify the targets of HDAC2 are warranted.

\section{Conclusions}

In summary, the current study demonstrated that chronic exposure of PM2.5 reprograms AM polarization toward the M2 activation state by reducing HDAC2 protein expression. This contributes to sustained airway inflammation, compromised lung function, emphysematous lesions, and deleterious airway remodeling. This study provides a new insight into the detrimental impact of chronic PM2.5 exposure in the development and progression of COPD and the underlying mechanism, thus presenting a novel avenue for treating this disease. 

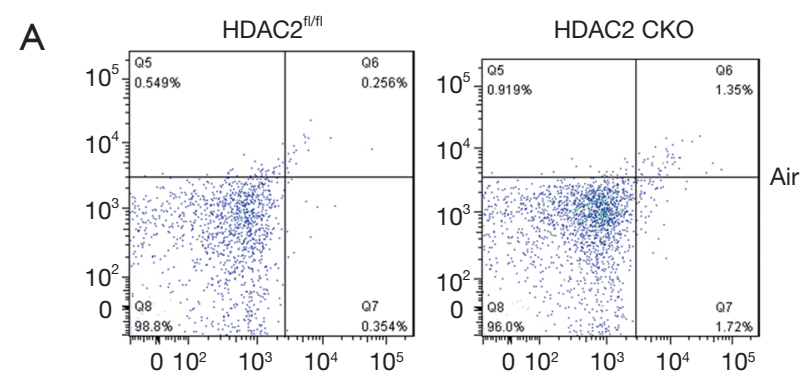

B
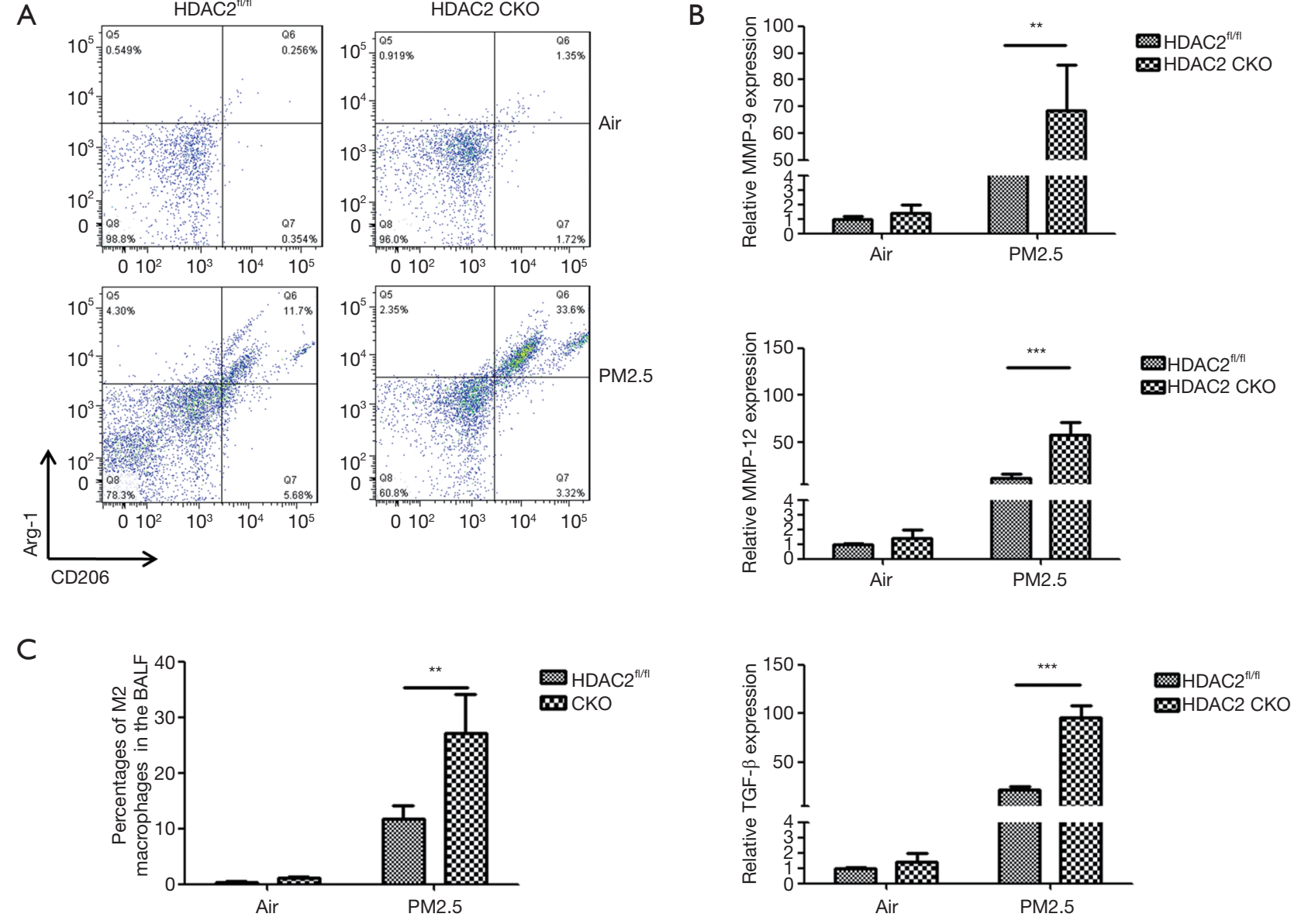

Figure 5 HDAC2 deficiency facilitated M2 macrophage polarization in a PM2.5-induced COPD model. (A) Representative FACS plots of M2 macrophages. (B) A statistical graph of FACS results. (C) The mRNA expression levels of MMP-9, MMP-12, and TGF- $\beta 1$. **, $\mathrm{P}<0.01$; *** $\mathrm{P}<0.001$. HDAC2, histone deacetylase 2; PM2.5, fine particulate matter; COPD, chronic obstructive pulmonary disease; FACS, fluorescence-activated cell sorting.

\section{Acknowledgments}

Funding: This work was supported by grants from the Natural Scientific Foundation of Shanghai (17140901201) and the National Natural Science Foundation of China (81972180, 81400056).

\section{Footnote}

Reporting Checklist: The authors have completed the ARRIVE reporting checklist. Available at http://dx.doi. org/10.21037/atm-20-6653

Data Sharing Statement: Available at http://dx.doi. org/10.21037/atm-20-6653
Conflicts of Interest: All authors have completed the ICMJE uniform disclosure form (available at http://dx.doi. org/10.21037/atm-20-6653). The authors have no conflicts of interest to declare.

Ethical Statement: The authors are accountable for all aspects of the work in ensuring that questions related to the accuracy or integrity of any part of the work are appropriately investigated and resolved. The study was approved by the Animal Care Committee of the Shanghai Pulmonary Hospital (No. K19-100Y) and were carried out in accordance with institutional conventions for the care and use of animals.

Open Access Statement: This is an Open Access article distributed in accordance with the Creative Commons 
Attribution-NonCommercial-NoDerivs 4.0 International License (CC BY-NC-ND 4.0), which permits the noncommercial replication and distribution of the article with the strict proviso that no changes or edits are made and the original work is properly cited (including links to both the formal publication through the relevant DOI and the license). See: https://creativecommons.org/licenses/by-nc-nd/4.0/.

\section{References}

1. Mathers CD, Loncar D. Projections of global mortality and burden of disease from 2002 to 2030. PLoS Med 2006;3:e442.

2. Agusti A, Zhang J. Chronic obstructive pulmonary disease at the beginning of the XXI Century. J Thorac Dis 2019;11:E210-3.

3. Song $\mathrm{C}, \mathrm{He} \mathrm{J}, \mathrm{Wu} \mathrm{L}$, et al. Health burden attributable to ambient PM2.5 in China. Environ Pollut 2017;223:575-86.

4. Gan WQ, FitzGerald JM, Carlsten C, et al. Associations of ambient air pollution with chronic obstructive pulmonary disease hospitalization and mortality. Am J Respir Crit Care Med 2013;187:721-7.

5. Guan WJ, Zheng XY, Chung KF, et al. Impact of air pollution on the burden of chronic respiratory diseases in China: time for urgent action. Lancet 2016;388:1939-51.

6. Sint T, Donohue JF, Ghio AJ. Ambient air pollution particles and the acute exacerbation of chronic obstructive pulmonary disease. Inhal Toxicol 2008;20:25-9.

7. Lepeule J, Laden F, Dockery D, et al. Chronic exposure to fine particles and mortality: an extended follow-up of the Harvard Six Cities study from 1974 to 2009. Environ Health Perspect 2012;120:965-70.

8. Dominici F, Peng RD, Bell ML, et al. Fine particulate air pollution and hospital admission for cardiovascular and respiratory diseases. JAMA 2006;295:1127-34.

9. Shen Y, Song J, Wang Y, et al. M2 macrophages promote pulmonary endothelial cells regeneration in sepsis-induced acute lung injury. Ann Transl Med 2019;7:142.

10. Byrne AJ, Mathie SA, Gregory LG, et al. Pulmonary macrophages: key players in the innate defence of the airways. Thorax 2015;70:1189-96.

11. Shapouri-Moghaddam A, Mohammadian S, Vazini H, et al. Macrophage plasticity, polarization, and function in health and disease. J Cell Physiol 2018;233:6425-40.

12. Sica A, Mantovani A. Macrophage plasticity and polarization: in vivo veritas. J Clin Invest 2012;122:787-95.

13. Mills CD. Anatomy of a discovery: $\mathrm{m} 1$ and $\mathrm{m} 2$ macrophages. Front Immunol 2015;6:212.
14. Rath M, Muller I, Kropf P, et al. Metabolism via Arginase or Nitric Oxide Synthase: Two Competing Arginine Pathways in Macrophages. Front Immunol 2014;5:532.

15. Mosser DM, Edwards JP. Exploring the full spectrum of macrophage activation. Nat Rev Immunol 2008;8:958-69.

16. Martinez FO, Gordon S. The M1 and M2 paradigm of macrophage activation: time for reassessment. F1000Prime Rep 2014;6:13.

17. Gordon S. Alternative activation of macrophages. Nat Rev Immunol 2003;3:23-35.

18. Vlahos R, Bozinovski S. Role of alveolar macrophages in chronic obstructive pulmonary disease. Front Immunol 2014;5:435.

19. Kuo $\mathrm{MH}$, Allis CD. Roles of histone acetyltransferases and deacetylases in gene regulation. Bioessays 1998;20:615-26.

20. Ito K, Ito M, Elliott WM, et al. Decreased histone deacetylase activity in chronic obstructive pulmonary disease. N Engl J Med 2005;352:1967-76.

21. Tan C, Xuan L, Cao S, et al. Decreased Histone Deacetylase 2 (HDAC2) in Peripheral Blood Monocytes (PBMCs) of COPD Patients. PLoS One 2016;11:e0147380.

22. Lopez-Campos JL, Tan W, Soriano JB. Global burden of COPD. Respirology 2016;21:14-23.

23. Huang D, Ma Z, He Y, et al. Long-term cigarette smoke exposure inhibits histone deacetylase 2 expression and enhances the nuclear factor-kappaB activation in skeletal muscle of mice. Oncotarget 2017;8:56726-36.

24. Barnes PJ. Role of HDAC2 in the pathophysiology of COPD. Annu Rev Physiol 2009;71:451-64.

25. Ito K, Barnes PJ, Adcock IM. Glucocorticoid receptor recruitment of histone deacetylase 2 inhibits interleukin1beta-induced histone $\mathrm{H} 4$ acetylation on lysines 8 and 12 . Mol Cell Biol 2000;20:6891-903.

26. Barnes PJ, Adcock IM. How do corticosteroids work in asthma? Ann Intern Med 2003;139:359-70.

27. Atkinson JJ, Senior RM. Matrix metalloproteinase-9 in lung remodeling. Am J Respir Cell Mol Biol 2003;28:12-24.

28. Takahashi T, Kubo H. The role of microparticles in chronic obstructive pulmonary disease. Int J Chron Obstruct Pulmon Dis 2014;9:303-14.

29. Border WA, Noble NA. Transforming growth factor beta in tissue fibrosis. N Engl J Med 1994;331:1286-92.

Cite this article as: Jiang Y, Zhao Y, Wang Q, Chen H, Zhou X. Fine particulate matter exposure promotes M2 macrophage polarization through inhibiting histone deacetylase 2 in the pathogenesis of chronic obstructive pulmonary disease. Ann Transl Med 2020;8(20):1303. doi: 10.21037/atm-20-6653 